Research Article

\title{
Formulation and Evaluation of Etoricoxib Niosomes by Thin Film Hydration Technique and Ether Injection Method
}

\author{
Veldurthi Ravalika, Abbaraju Krishna Sailaja \\ Department of pharmaceutics, RBVRR Women's College of Pharmacy, Barkatpura, Hyderabad, India. \\ Corresponding author. E-mail: shailaja1234@rediffmail.com
}

Received: Aug. 3, 2017; Accepted: Sep. 28, 2017; Published: Sep. 30, 2017.

Citation: Veldurthi Ravalika, Abbaraju Krishna Sailaja, Formulation and Evaluation of Etoricoxib Niosomes by Thin Film Hydration Technique and Ether Injection Method. Nano Biomed. Eng., 2017, 9(3): 242-248.

DOI: $10.5101 /$ nbe.v9i3.p242-248.

\begin{abstract}
The main objective of this study is to formulate etoricoxib niosomes as vesicular carriers for site specific drug delivery. Niosomes are novel vesicular carriers, in which the drug is incorporated in a vesicle. Niosomal vesicles are formed by hydrating mixture of cholesterol and nonionic surfactants. Niosomes can increase the permeability of the skin (stratum corneum and epidermis), by avoiding the first pass metabolism and also reduce the side effects. Etoricoxib is a potent new COX-2 inhibitor used in the treatment of osteoarthritis, rheumatoid arthritis, ankylosing spondylitis, acute gout arthritis etc. Two formulations were prepared by thin film hydration technique using the drug, cholesterol and surfactants Tween 80 (F1) and Span 60 (F2). Another two formulations were prepared by ether injection method using cholesterol and surfactants Tween 80 (F3) and Span 60 (F4). Each formulation was evaluated for drug content, entrapment efficiency, mean vesicular diameter, zeta potential and In-vitro drug release studies. Among the four formulations, F2 formulation containing the drug and Span 60 showed maximal drug content of $95.57 \%$, entrapment efficiency of $96.40 \%$, mean vesicular diameter of $463.7 \mathrm{~nm}$, zeta potential of $-80.5 \mathrm{mV}$, in-vitro drug release of $95.14 \%$ in $12 \mathrm{~h}$, and the drug release followed the first order with non-fickian diffusion mechanism by thin film hydration technique. Hence, the thin film hydration technique is an optimized technique for the preparation of etoricoxib niosomes.
\end{abstract}

Keywords: Niosomes; Cholesterol; Span 60; Tween 80; Etoricoxib; Entrapment efficiency

\section{Introduction}

Niosomes are one of the promising drug carriers that have a bilayer structure and are formed by selfassociation of nonionic surfactants and cholesterol in an aqueous phase (Fig. 1) [7,8]. Niosomes are alternative carriers to liposomes, usually made up of synthetic, non ionic surfactant of alkyl or di-alkyl poly glycerol ether class. The uni- or multilamellar niosomes can entrap solutes and have more stable in-vitro release and can improve the stability and duration of action of entrapped drug as compared to the stability of conventional dosage forms.

\section{Advantages of niosomes}

Niosomes are osmotically active and stable, and also they increase the stability of entrapped drug. Nisomes improve oral bioavailability of poorly absorbed drugs and enhance skin penetration of drugs; they can be made to reach the site of action via oral, parenteral 


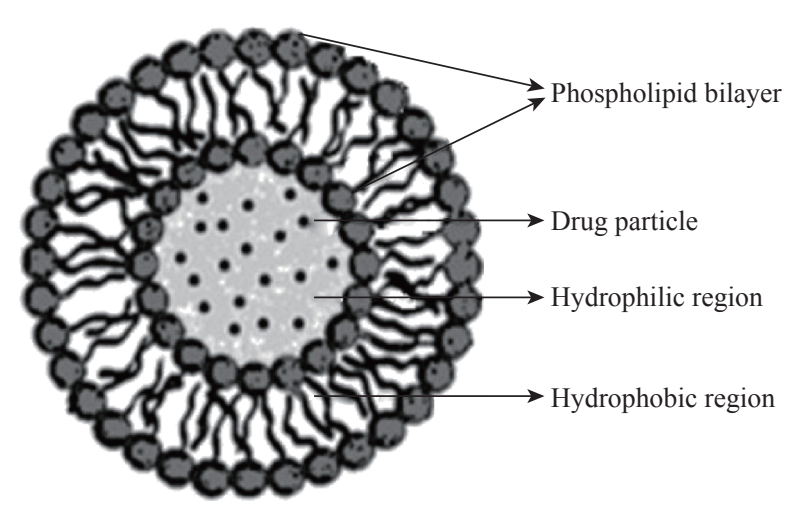

Fig. 1 Structure of niosome.

as well as topical routes. Niosomes containing the surfactants are biodegradable, biocompatible and nonimmunogenic. Niosomes improve the therapeutic performance of the drug molecules by delayed clearance from the circulation, protecting the drug from biological environment and restricting effects to target cells $[1,2]$.

\section{Applications of niosomes}

Niosomal drug delivery has a number of potential advantages over conventional dosage forms. Niosomes are potentially applicable to many pharmacological agents for their action against various diseases. An increase in the penetration rate has been achieved by transdermal delivery of drugs incorporated in niosomes. The incorporation of drugs with low therapeutic index and low water solubility will sustain the drug release action of niosomes; the localized drug action of niosomes results in enhancement of efficacy of potency of the drug and reduces its systemic toxic effects. Niosomes can be used as a carrier for hemoglobin and also used for studying the nature of the immune response provoked by antigen $[3,5]$.

Etoricoxib (5-chloro-2-(6-methylpyridin-3-yl)3-(4-methylsulfonylphenyl) pyridine) is a novel orally active agent that selectively inhibits $\mathrm{COX}-2$ (cyclooxygenase-2). Etoricoxib is used in the treatment of rheumatoid arthritis, osteoarthritis, acute gout, chronic musculoskeletal pain (including chronic low back pain), postoperative dental pain and primary dysmenorrhoea. The recommended dosage of the drug is between 60 and $120 \mathrm{mg}$ /day as oral tablets. It is a poorly soluble, lipophilic dug with estimated $\log \mathrm{P}$ of 3.14 and $\mathrm{pKa}$ of 4.6. Its aqueous solubility is low and highly $\mathrm{pH}$-dependent. Etoricoxib is completely and rapidly absorbed, with an oral bioavailability of up to $100 \%[16]$.

The purpose of this work is to formulate etoricoxib niosomes as carriers to reduce the risk of side effects and to achieve the localized action. Etoricoxib is available as tablets, capsules and emulgels. The long term use of this drug, when taken as oral formulations leads to severe adverse drug reactions such as constipation, diarrhoea, nausea, vomiting, oesophagitis, flatulence, gastritis, heartburn/acid reflux, dyspepsia/epigastric discomfort, oral ulcer, alveolar osteitis, oedema/fluid retention, dizziness, headache, palpitations, arrhythmia, HTN, asthenia/fatigue, flu-like disease, bronchospasm, Stevens-Johnson syndrome, exfoliative dermatitis and toxic epidermal necrolysis, upper gastrointestinal (GI) ulceration, perforation and bleeding. Hence there is a need to develop site specific drug delivery system to avoid side effects of etoricoxib [16].

\section{Experimental}

\section{Materials}

Etoricoxib was purchased from Hetero Chemicals Pvt. Limited, Hyderabad. Cholesterol, Span 60, Tween 80, chloroform, ethanol, $\mathrm{pH} 7.4$ buffer, diethyl ether and methanol were from SD Fine-Chem. Limited, Mumbai.

\section{Methods of preparation of niosomes Thin film hydration technique}

The mixture of vesicles forming ingredients like surfactant and cholesterol were dissolved in a volatile organic solvents chloroform and ethanol (1:2) in a round bottom flask. The organic solvent was then removed above the lipid transition temperature by using rotary evaporator, leaving a thin layer of solid mixture deposited on the wall of the flask. The dried surfactant film could be rehydrated with $10 \mathrm{~mL}$ of aqueous phase ( $\mathrm{pH} 7.4$ buffer) at $0-60{ }^{\circ} \mathrm{C}$ with gentle agitation. This process formed typical multilamellar niosomes (Table 1) [4, 5].

\section{Ether injection method}

This method provided a means of making niosomes by slowly introducing nonionic surfactant and cholesterol dissolved in diethyl ether mixed with 2 $\mathrm{mL}$ methanol previously containing weighed quantity of drug. The resulting solution was slowly injected using microsyringe into $10 \mathrm{~mL}$ hydrating phosphate buffer at a rate of $1 \mathrm{~mL} / \mathrm{min}$ on magnetic stirrer, and the temperature maintained at $60-65^{\circ} \mathrm{C}$. Then the lipid solution was injected slowly into aqueous 
Table 1 Formulations for thin film hydration technique

\begin{tabular}{cccc}
\hline $\begin{array}{c}\text { Formulation } \\
\text { code }\end{array}$ & Surfactant & Method of preparation & $\begin{array}{c}\text { Drug : surfactant : } \\
\text { cholesterol }\end{array}$ \\
\hline F1 & Tween 80 & Thin film hydration & $1: 1: 1$ \\
F2 & Span 60 & Thin film hydration & $1: 1: 1$ \\
\hline
\end{tabular}

Table 2 Formulations for ether injection method

\begin{tabular}{cccc}
\hline $\begin{array}{c}\text { Formulation } \\
\text { code }\end{array}$ & Surfactant & $\begin{array}{c}\text { Method of } \\
\text { preparation }\end{array}$ & $\begin{array}{c}\text { Drug : surfactant : } \\
\text { cholesterol }\end{array}$ \\
\hline F3 & Tween 80 & Ether injection & $1: 1: 1$ \\
F4 & Span 60 & Ether injection & $1: 1: 1$ \\
\hline
\end{tabular}
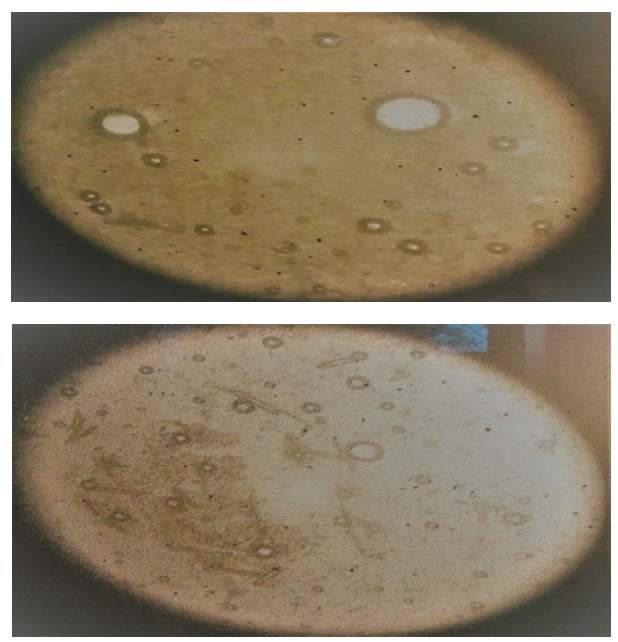

Fig. 2 Optical images of niosomal formultion.

phase. Differences in temperature between the phases caused rapid vapourization of ether and resulted in the formation of niosomal vesicles (Table 2) [11, 12].

\section{Charecterization of niosomes Microscopic evaluation of niosomes}

One drop of niosomal dispersion was taken on the glass slide and observed under projection microscope with $10 \times$ magnification (Fig. 2).

\section{Mean vesicular diameter}

A small amount of optimized niosomal formulation was mixed with $5 \mathrm{~mL}$ double distilled water and kept for $1 \mathrm{~h}$ sonication. Then the sample was analyzed by using nanoparticle analyzer (HORIBA Nanopartica SZ-100) for particle size determination $[10,13]$.

\section{Zeta potential}

The optimized formulation was analyzed for zeta potential value to determine the stability of the formulation. The analysis was carried out by using double distilled water as dispersion medium at the temperature of $25^{\circ} \mathrm{C}[11,14]$.

\section{Drug content}

Niosomal suspension equivalent to $5 \mathrm{mg}$ was taken in a $10 \mathrm{~mL}$ volumetric flask, and the volume was made with $7.4 \mathrm{pH}$ buffer to disrupt the vesicle by sonication for $40 \mathrm{~min} .1 \mathrm{~mL}$ of the solution was pipetted out in $50 \mathrm{~mL}$ volumetric flask; the volume was made with buffer and kept for sonication. Then the concentration of drug was analyzed by UV spectrophotometer at $235 \mathrm{~nm}[15,17]$.

\section{Entrapment efficiency}

After preparing niosomal dispersion, unentrapped drug was separated by centrifugation using $\mathrm{pH}$ 7.4 phosphate buffer for $45 \mathrm{~min}$ at $17,000 \mathrm{rpm}$. The resulting solution was analyzed by UV spectrophotometer at $235 \mathrm{~nm}$ for the total amount of entrapped drug $[9,13]$.

Entrapment efficiency $=$

$\frac{\text { Amount of drug taken }- \text { amount of drug in supernatant }}{\text { amount of drug }} \times 100$

\section{In-vitro drug release studies}

In-vitro drug release studies of transferosomes were carried out by Franz diffusion cell. $1 \mathrm{~mL}$ of niosomal formulation was placed on cellophane membrane between the donor compartment and the receptor compartment containing $50 \mathrm{~mL}$ of $\mathrm{pH} 7.4$ buffer. The samples were withdrawn using microsyringe at particular interval of time with replacing fresh buffer to maintain the sink condition. The absorbance of each sample was analyzed by UV spectrophotometer at 235 $\mathrm{nm}$, and the in-vitro drug release was calculated $[18,19]$.

\section{Results and Discussion \\ Comparison of drug content of niosomal formulation prepared by both the techniques}

The prepared niosomal formulations F1, F2, F3 and F4 were evaluated for drug content. As shown in Table 3, the percentage of drug content was found to be $94.96 \%, 95.57 \%, 89.02 \%$ and $93.12 \%$ respectively. Among the four formulations, F2 showed maximal drug content as of $95.57 \%$ (Fig. 3).

Table 3 Percentage of drug content of niosomal formulations

\begin{tabular}{cc}
\hline Formulation code & Percentage of drug content \\
\hline F1 & $94.96 \%$ \\
F2 & $95.57 \%$ \\
F3 & $89.02 \%$ \\
F4 & $93.12 \%$ \\
\hline
\end{tabular}




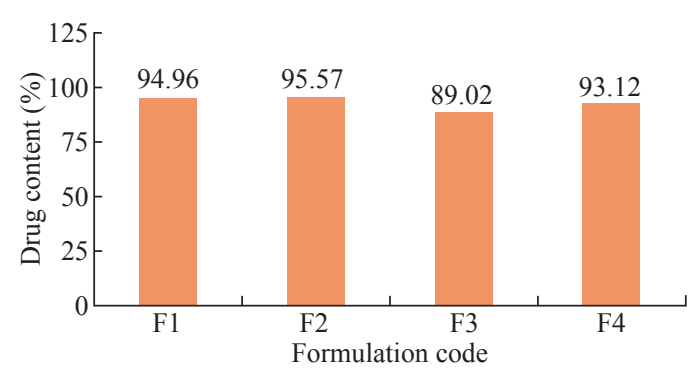

Fig. 3 Comparison of drug content of niosomal formulations.

\section{Comparision of entrapment efficiency of niosomes prepared by ether injection method and thin film hydration technique}

The prepared niosomal formulations F1, F2, F3 and F4 were evaluated for entrapment efficiency. As shown in Table 4, the percentage of entrapment efficiency was found to be $92.31 \%, 96.4 \%, 84.51 \%$ and $93.23 \%$ respectively. Among the four formulations, F2 showed maximal entrapment efficiency as of $96.4 \%$ (Fig. 4).

\section{Mean vesicular diameter}

The mean vesicular diameter of all formulations was

Table 4 Percentage of entrapment efficiency of niosomal formulations

\begin{tabular}{cc}
\hline Formulation code & Entrapment efficiency \\
\hline F1 & $92.31 \%$ \\
F2 & $96.40 \%$ \\
F3 & $84.51 \%$ \\
F4 & $93.23 \%$ \\
\hline
\end{tabular}

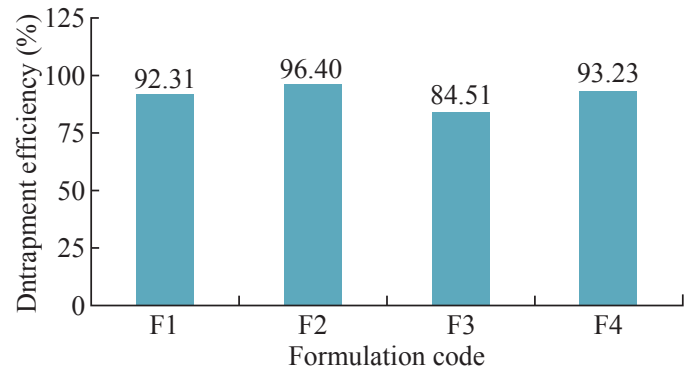

Fig. 4 Comparison of entrapment efficiency of niosomal formulations.

evaluated by using HORIBA Nanopartica. Among the four formulations, F2 (with Span 60) showed a mean vesicular diameter of $463.7 \mathrm{~nm}$ (Fig. 5).

\section{Zeta potential determination}

The zeta potential of the all formulations was carried out by using zeta sizer. Among all formulations, F2 formulation was found to be $-80 \mathrm{mV}$ which indicated the formulation was highly stable (Fig. 6).

\section{In-vitro diffusion study}

The in-vitro drug release studies for all formulations were performed. The optimized formulation showed the drug release of $95.14 \%$ within $12 \mathrm{~h}$ (Fig. 7).

The prepared niosomal formulations F1, F2, F3 and F4 were subjected to in-vitro diffusion studies. The percentage of drug release was found to be $90.97 \%$, $95.14 \%, 43.62 \%$ and $29.88 \%$ respectively. Among all four formulations, F2 showed maximal drug release as of $95.14 \%$ in $12 \mathrm{~h}$.

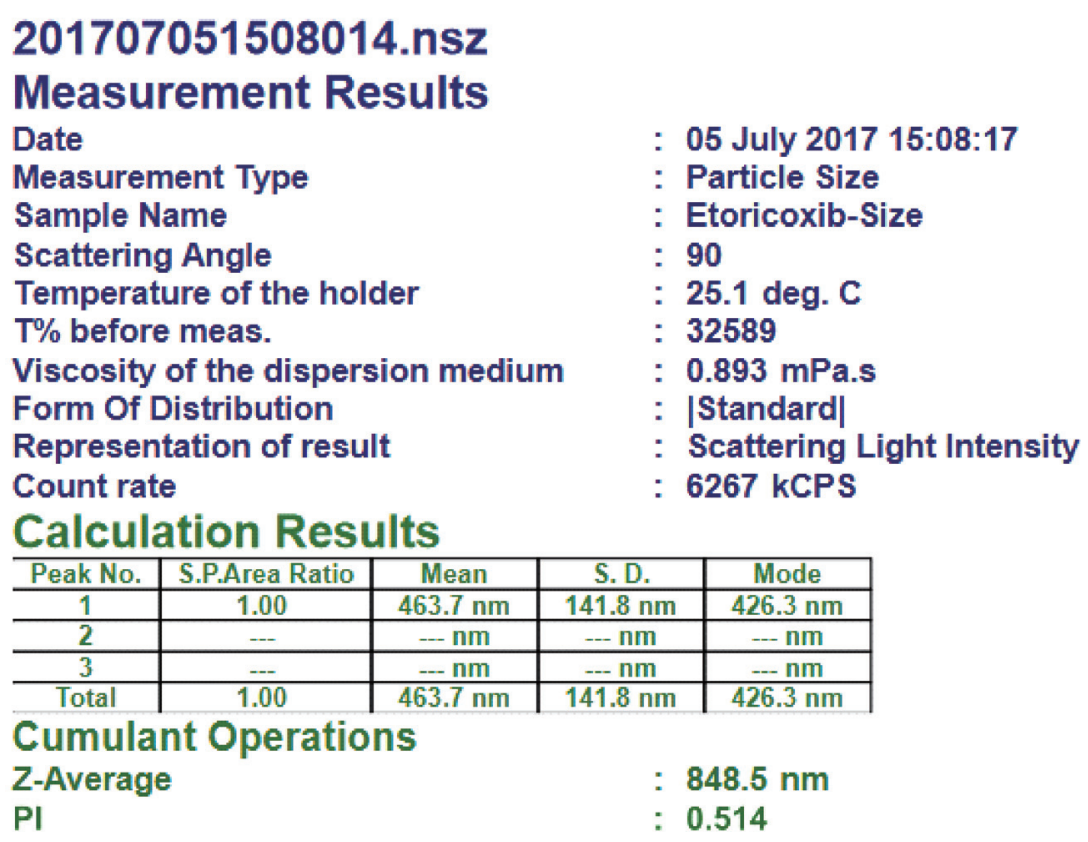

Fig. 5 Mean vesicular diameter of formulation F2 prepared by thin film hydration technique using surfactant Span 60. 


\section{Measurement Results}

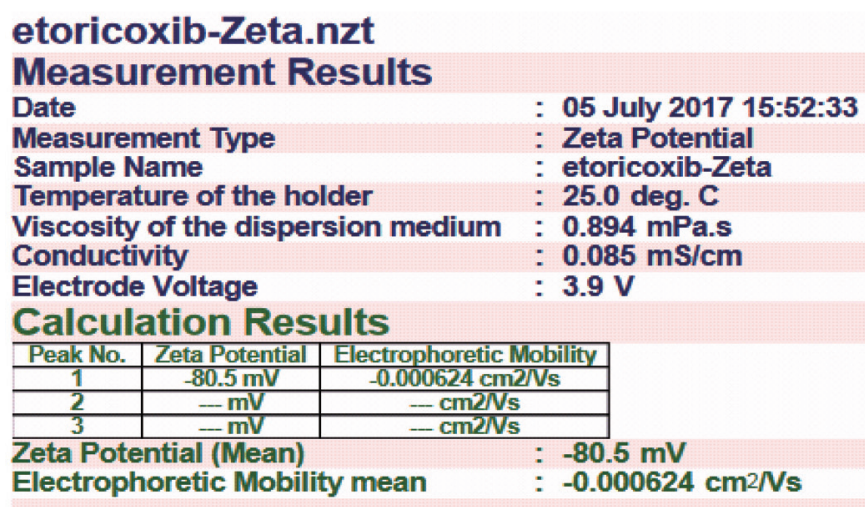

Fig. 6 Zeta potential value for formulation F2 prepared by thin film hydration technique using surfactant Span 60 .

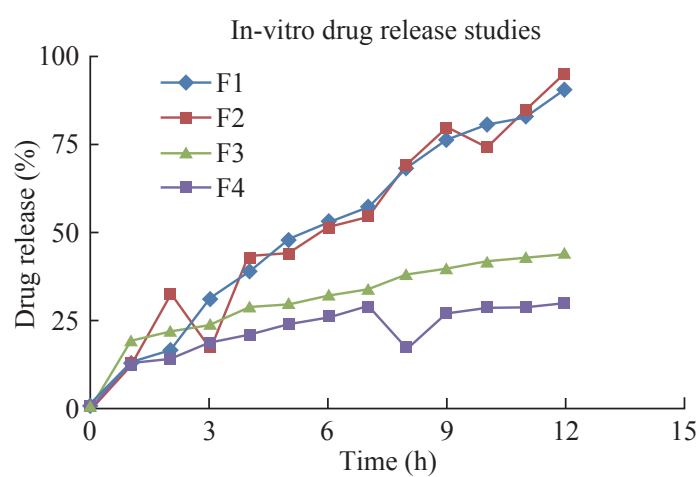

Fig. 7 In-vitro drug release for four formulations F1, F2, F3 and $\mathrm{F} 4$ as prepared by thin film hydration technique and ether injection method.
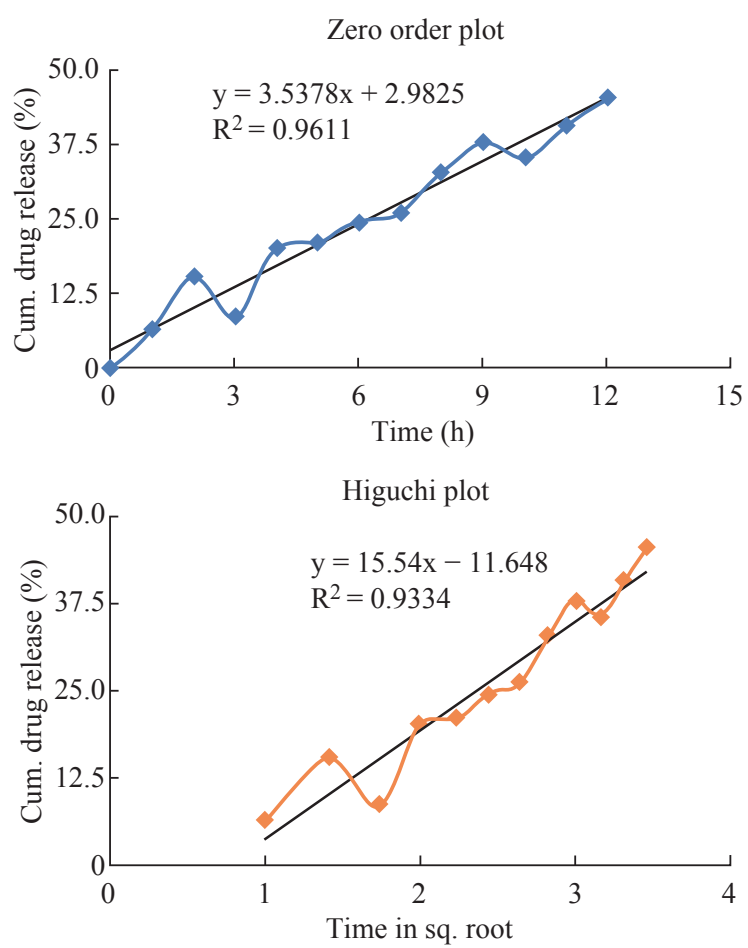

\section{Kinetics of drug release}

Zero order, first order, Higuchi and KorsmeyerPeppas plots were drawn for the optimized formulation $\mathrm{F} 2$, in order to demonstrate the release kinetics of the drug (Fig. 8) [4, 11]. According to the kinetic plots, the formulation was following first order release with nonfickian diffusion.

The four formulations of etoricoxib niosomes were prepared by thin film hydration technique and ether injection method using surfactants including Span 60 and Tween 80 and by adding equal amount of cholesterol and drug. Among all formulations,
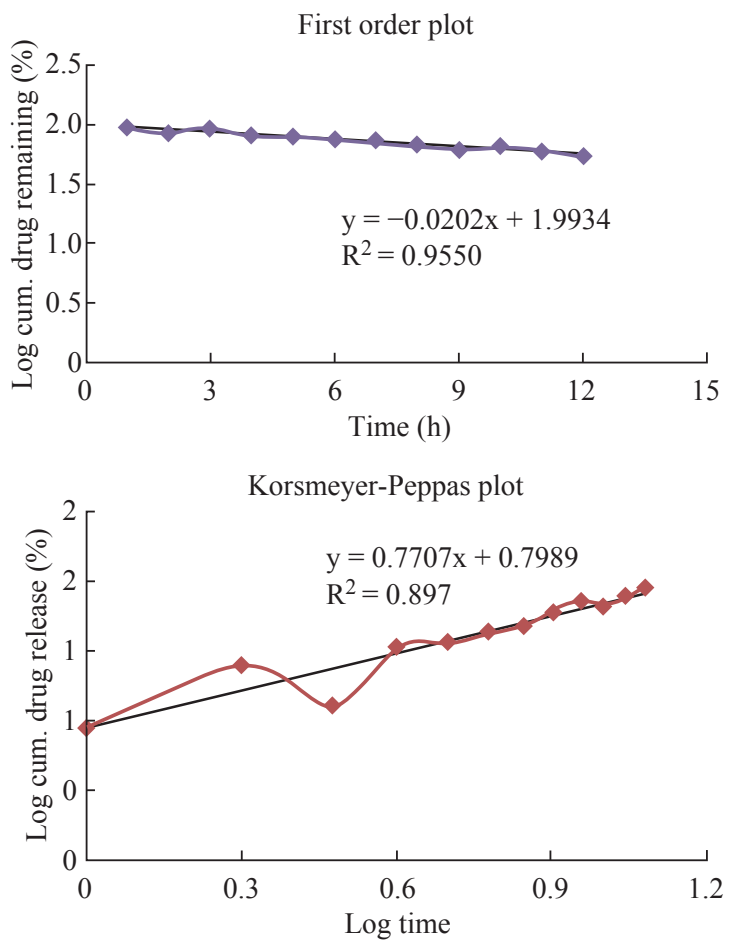

Fig. 8 Kinetic release data for optimized formulation F2. 
Table 5 Kinetic release data for optimized formulation F2

\begin{tabular}{ccccc}
\hline Formulation & Zero order $\left(\mathrm{R}^{2}\right)$ & First order $\left(\mathrm{R}^{2}\right)$ & Higuchi plot $\left(\mathrm{R}^{2}\right)$ & Peppas plot $(\mathrm{n})$ \\
\hline $\mathrm{F} 2$ & 0.947 & 0.955 & 0.933 & 0.770 \\
\hline
\end{tabular}

formulation F2 showed the maximal drug content, entrapment efficiency and mean vesicular diameter.

Previously, works have been done for the preparation of niosomes containing etodolac for topical drug delivery using cholesterol and Span 60 by thin film hydration technique, which showed good entrapment efficiency as of $96.72 \%$ and drug release as of $94.91 \%$ after $24 \mathrm{~h}$ [19].

Rahman et al. developed curcumin loaded niosomal transdermal gel using Span 60 and cholesterol in the ratio of $7: 3 \mathrm{mmol}$. After evaluation, it was found that the formulation containing Span 60 showd better entrapment efficiency as of $61.22 \%$ and particle size as of 1-5 $\mu \mathrm{m}$ [20].

Diclofenac sodium niosomes were developed by using Span 20, 40, 60, 80, 85 and Tween 20, 40, 60, 80, 85 and by hand-shaking method. Among all formulations, the formulation containing Span 60 showed better entrapment efficiency as of $30.52 \%[21,22]$.

These works proved that Span 60 was used as a good surfactant for the formation of niosomes, and the thin film hydration technique was a better technique to formulate good nisomal vesicles. Hence, the thin film hydration technique was selected for the preparation of etoricoxib niosomes [20, 21].

Usually, surfactant with a hydrophilic-lipophilic balance (HLB) value between 4 to 8 is mostly preferred for the vesicle formation. Increasing the HLB value will moderately decrease the entrapment efficiency, vesicle formation and also influence the stability of the drug. The HLB value of sorbitan monostearate Span 60 is 4.7 , and it is solid at room temperature, having the highest phase transition temperature of $50{ }^{\circ} \mathrm{C}$ [12]. The surfactant has the highest transition temperature and produces the highest entrapment efficiency. Hence, the formulation F2 containing Span 60, cholesterol and drug showed entrapment efficiency as of $96.40 \%$, drug content as of $95.57 \%$, mean vesicular diameter as of $463.7 \mathrm{~nm}$ and zeta potential as of $-80.5 \mathrm{mv}$. The drug release was found to be $95.14 \%$ in $12 \mathrm{~h}$. The optimized formulation followed the first order kinetics with nonfickian diffusion mechanism.

\section{Conclusions}

Etoricoxib niosomes were successfully developed as a carrier for site-specific drug delivery by thin film hydration technique using the drug, cholesterol and surfactants. The formulation containing surfactant Span 60 showed maximal drug content as of $95.57 \%$, better entrapment efficiency as of $96.40 \%$, minimal mean vesicular diameter as of $463.7 \mathrm{~nm}$, and zeta potential as of $-80.5 \mathrm{mV}$. Hence, Span 60 was used as a good surfactant to form niosomes and thin film hydration technique was an optimized technique for the preparation of etoricoxib niosomes.

\section{Conflict of Interests}

The authors declare that no competing interest exists.

\section{References}

[1] M. Chauhan, S. Sharma, Span 60 niosomal oral suspension of fluconazole: Formulation and in vitro evaluation. JPRHC, 2009, 1: 142-156.

[2] V. Shakya, B.K. Bansal, Niosomes: A novel trend in drug delivery. Int. J. Res. Dev. Pharm. L. Sci, 2014, 3(4): 10361041.

[3] Y.P. Kumar, K.V. Kumar, Formulation and Evaluation of econozole niosomes, Sch. Acad. J. Pharm., 2013, 2(4): 315-318.

[4] S. Kamboj, V. Saini, S. Bala, et al., Formulation and characterization of drug loaded niosomal gel for antiinflammatory activity, International Journal of Medical, Health, Pharmaceutical and Biomedical Engineering, 7(12), 2013, 541-545.

[5] B.V. Lokeswara, S.T. Mathew, K.N. Jayaveera, et al., Niosme-vesicular drug delivery system. International Journal of Pharmacy, 2013, 3(1): 180-185.

[6] P. Balakrishnan, S. Shanmuga, W.S. Lee, et al., Formulation and in vitro assessment of minoxidil niosomes for enhanced skin delivery. Int. J. Pharm., 2009, 377(1): 1-8.

[7] I.P. Kaur, A. Garg, A.K. Singla, et al., Vesicular systems in ocular delivery: an overview. Int. J. Pharm., 2004, 269: $1-14$.

[8] I.F. Uchegbu, S.P. Vyas. Non ionic surfactant based vesicles (niosomes) in drug delivery. Int. J. Pharm., 1998, 172: 33-70.

[9] R.A. Naresh, G.K. Pillai, N. Udupa, et al., Antiinflammatory activity of niosome encapsulated diclofenac sodium in arthritic rats. Indian. J. Pharmacol., 1994, 26: 46-48.

[10] A. Solankia, Preparation, characterization, optimization, and stability studies of aceclofenac proniosomes. Iranian Journal of Pharmaceutical Research, 2008, 7(4): 237246.

[11] P. Sundaresan, Evaluation of aceclofenac niosomes prepared by various techniques. International Journal of Pharmaceutical Sciences Review and Research, 2012, 
16(1): 75-78.

[12] A. Gadhave, Determination of hydrophilis-lipophilic balance value. International Journal of Science and Reaserch, 2014: 573-575.

[13] F. Szoka Jr., D. Papahadjopoulos, Comparative Properties and methods of preparation of lipid vesicles (liposomes). Annual review of Biophysics andBbioengineering, 1980, 9: 9467-9508.

[14] O. Naksuriya, S. Okonogi, Comparison and combination effects on antioxidant power of curcumin with gallic acid, ascorbic acid, and xanthone. Drug Discoveries \& Therapeutics, 2015, 9(2): 136-141.

[15] V.R. Gupta, S.Mutalik, M.M.Patel, et al., Spherical crystals of celecoxib to improve solubility, dissolution rate and micromeritic properties, Acta Pharm.,2007, 57: 173-184.

[16] R. Sofia. Update on the clinical pharmacology of etoricoxib, a potent cyclooxygenase- 2 inhibitor. Future Rheumatol., 2007, 2(6): 545-565.

[17] P. Moser, M. Marchand-Arvier, P. Labrude, et al., Niosomes d'hémoglobine. I. Preparation, proprietes physicochimiques et oxyphoriques, stabilite. Pharma. Acta. Helv., 1989, 64 (7): 192-202.

[18] M.N. Azmin, A.T. Florence, R.M. Handjani-Vila, et al., The effect of nonionic surfactant vesicle (niosome) entrapment on the absorption and distribution of methotrexate in mice. J Pharm Pharmacol, 1985, 37: 237-242.

[19] R. Supraja, A.K. Sailaja, Formulation of mefenamic acid loaded ethosomal gel by hot and cold methods. Nano Biomed. Eng., 2017, 9(1): 27-35

[20] L. Rahman, Arisanty, and M.A. Manggau. Niosomal transdermal gel formulation of curcumin having antiinflammatory effect in experimental rat models. Journal of Chemical and Pharmaceutical Reaserch, 2015, 7(9): 843-849.

[21] M. Abdallah, O. Sammour, H. EL-Ghamry, et al., Preparation and In-vitro Evaluation of Diclofenac Sodium Niosomal Formulatuion. IJPSR, 2013, 4(5): 1757-1765.

[22] S.M.M. Al-Mutoki, B.A.K. Al-Ghzawi, E.A.J. Al-Mulla,, et al. Enhancement of mechanical properties of polyamide hexaglycol by dispersion of $\mathrm{TiO} 2$ nanofiller. Nano Biomed. Eng., 2016, 8(2): 55-59.

Copyright (C) Veldurthi Ravalika, Abbaraju Krishna Sailaja. This is an open-access article distributed under the terms of the Creative Commons Attribution License, which permits unrestricted use, distribution, and reproduction in any medium, provided the original author and source are credited. 